\title{
Quantum Transport: The Link between Standard Approaches in Superlattices
}

\author{
Wacker, Andreas; Jauho, Antti-Pekka
}

Published in:

Physical Review Letters

Link to article, DOI:

10.1103/PhysRevLett.80.369

Publication date:

1998

Document Version

Publisher's PDF, also known as Version of record

Link back to DTU Orbit

Citation (APA):

Wacker, A., \& Jauho, A-P. (1998). Quantum Transport: The Link between Standard Approaches in Superlattices. Physical Review Letters, 80(2), 369-372. https://doi.org/10.1103/PhysRevLett.80.369

\section{General rights}

Copyright and moral rights for the publications made accessible in the public portal are retained by the authors and/or other copyright owners and it is a condition of accessing publications that users recognise and abide by the legal requirements associated with these rights.

- Users may download and print one copy of any publication from the public portal for the purpose of private study or research.

- You may not further distribute the material or use it for any profit-making activity or commercial gain

- You may freely distribute the URL identifying the publication in the public portal

If you believe that this document breaches copyright please contact us providing details, and we will remove access to the work immediately and investigate your claim. 


\title{
Quantum Transport: The Link between Standard Approaches in Superlattices
}

\author{
Andreas Wacker* and Antti-Pekka Jauho \\ Mikroelektronik Centret, Bldg 345 east, Danmarks Tekniske Universitet, 2800 Lyngby, Denmark
}

(Received 25 September 1997)

\begin{abstract}
Theories describing electrical transport in semiconductor superlattices can essentially be divided in three disjoint categories: (i) transport in a miniband; (ii) hopping between Wannier-Stark ladders; and (iii) sequential tunneling. We present a quantum transport model, based on nonequilibrium Green functions, which, in the appropriate limits, reproduces the three conventional theories and describes the transport in the previously inaccessible region of the parameter space. [S0031-9007(97)04960-0]
\end{abstract}

PACS numbers: 73.61.-r, 72.10.-d, 72.20.Ht

Ever since the pioneering work of Esaki and Tsu [1], which drew attention to the rich physics and potential device applications of semiconductor superlattices, these man-made structures have remained a topic of intense research. Semiconductor superlattices have proven to be a fruitful platform for studying a wide range of transport phenomena, such as their intrinsic negative differential conductivity [2], the formation of electric field domains [3], Bloch oscillations [4], as well as dynamical localization [5] and absolute negative conductance [6] under external irradiation, just to mention a few.

These phenomena depend crucially on the relations of the energy scales involved, namely, the zero-field miniband width (which is 4 times the interwell coupling $T_{1}$ ), the scattering rate $\Gamma / \hbar$, and the potential drop per pe$\operatorname{riod}$ ( $\equiv e F d$, where $F$ is the applied static field and $d$ is the superlattice period). Three distinct approaches have been used to describe transport in the parameter space spanned by $\left(T_{1}, e F d, \Gamma\right)$ : miniband conduction (MBC) [1,7], Wannier-Stark hopping (WSH) [8], and sequential tunneling (ST) $[9,10]$. While the ranges of validity of the different approaches have been addressed qualitatively before [11-14], no explicit calculations have been presented where the different ranges can be identified and the transition between them can be studied. In the present Letter we present such a calculation, based on nonequilibrium Green functions. The calculated current-field relations are shown to reflect the results from the simple approaches (MBC, WSH, and ST, which will be reviewed below) in their respective ranges of validity sketched in the "phase diagram" presented in Fig. 1. While similar diagrams have been obtained in Refs. [13,14] from more phenomenological arguments, we will derive the borderlines from our Green function analysis here.

Now, we introduce the model assumptions which will be used in each of the following approaches. We restrict ourselves to the lowest miniband of the superlattice. Our basis sets are orthonormal wave functions $\Psi_{n}(z) e^{i(\mathbf{k} \cdot \mathbf{r})} / A$ where the $z$ direction denotes the growth direction. The $\Psi_{n}(z)=\Psi(z-n d)$ are localized in well $n$ (for example, one may use the Wannier functions). Here $\mathbf{r}$ and $\mathbf{k}$ denote two-dimensional vectors within the $(x, y)$ plane (with area $A$ ) which is assumed to be separable from the $z$ direction.
For parabolic dispersion $E_{k}=\hbar^{2} \mathbf{k}^{2} / 2 m$ (with the effective mass $m$ of the conduction band) we thus have a constant density of states $\rho_{0}=m / \pi \hbar^{2}$ per area and period. The single-particle part of the Hamiltonian within nearest neighbor coupling is then given by

$$
\hat{H}_{n, m}^{\mathrm{SL}}=\left(\delta_{n, m-1}+\delta_{n, m+1}\right) T_{1}+\delta_{n, m}\left(E_{k}-n e F d\right) .
$$

Furthermore we consider a phenomenological scattering process at $\delta$ potentials with density $N_{d}$ and matrix element $\delta_{n, m} V / A$, leading to a scattering rate $1 / \tau_{0}=\Gamma_{0} / \hbar=$ $N_{d} \pi V^{2} \rho_{0} / \hbar$ between the $\mathbf{k}$ states within a given well. Finally, we assume that the inscattering term is determined by a Fermi distribution $n_{F}(E)=\{1+\exp [(E-$ $\left.\left.\mu) / k_{B} T_{e}\right]\right\}^{-1}$ with electron temperature $T_{e}$ and chemical potential $\mu$. This assumption establishes internal energy relaxation without specifying the corresponding processes in detail. It has been implicitly used in the standard approaches $[7,8,10]$ as well.

Miniband conduction. - For zero electric field Eq. (1) is diagonalized by a set of Bloch functions $\varphi_{q}(z)=$ $\sum_{n} e^{i n q d} \Psi_{n}(z)$ and the dispersion relation is given by the

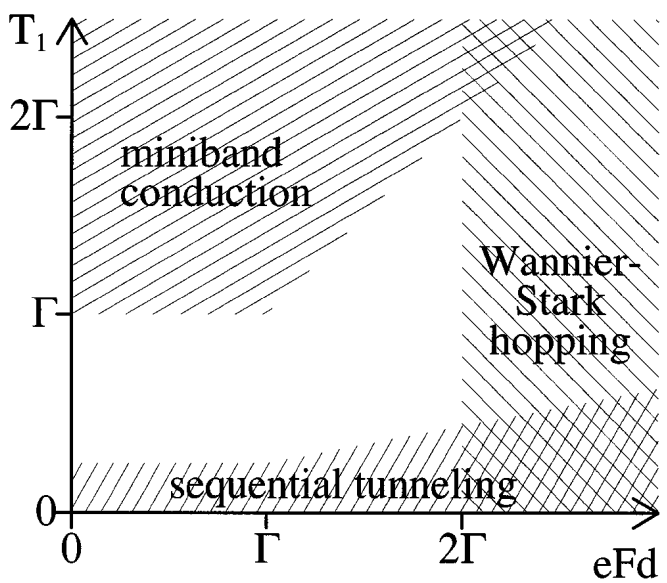

FIG. 1. Regimes where the different transport models are valid for low electron densities and low temperatures. (For illustrative purpose we have translated the condition $a \gg b$ in the text to $a>2 b$, where $a$ and $b$ denote the respective energy scales involved.) 
miniband $E(q)=2 T_{1} \cos (q d)$. The stationary Boltzmann equation for the distribution function $f(q, \mathbf{k})$ is then

$$
\frac{e F}{\hbar} \frac{\partial f(q, \mathbf{k})}{\partial q}=\frac{n_{F}\left[E(q)+E_{k}\right]-f(q, \mathbf{k})}{\tau\left[E(q)+E_{k}\right]},
$$

where the relaxation-time approximation corresponds to our assumption on scattering mentioned above. For our scattering model, we obtain the relaxation time $\tau(E)=\tau_{0}$ for $E \geq 2\left|T_{1}\right|$ and $\tau(E)=\pi \tau_{0} / \arccos \left(-E / 2\left|T_{1}\right|\right)$ for $-2\left|T_{1}\right| \leq E<2\left|T_{1}\right|$. Equation (2) is solved numerically and the current is calculated from

$$
J(F)=\frac{e}{4 \pi^{3} \hbar} \int d^{2} k \int_{-\pi / d}^{\pi / d} d q f(q, \mathbf{k}) \frac{d E(q)}{d q} .
$$

The electron density per period is given by

$$
N_{2 \mathrm{D}}=\frac{d}{4 \pi^{3}} \int d^{2} k \int_{-\pi / d}^{\pi / d} d q f(q, \mathbf{k})
$$

and is used to determine the chemical potential [which is field dependent due to the energy dependence of $\tau(E)$ ] for a given electron density. This approach can be extended beyond the relaxation-time approximation $[15,16]$, but the generic features remain unchanged.

Wannier-Stark hopping. - In the presence of an electric field, the eigenstates of the Hamiltonian become the localized Wannier-Stark states,

$$
\phi_{\nu}(z)=\sum_{n} J_{n-\nu}\left(\frac{2 T_{1}}{e F d}\right) \Psi_{n}(z),
$$

with energy $E_{\nu}=-\nu e F d$ where $J_{n}(z)$ is the Bessel function of the first kind. Scattering causes hopping between the different states. Within Fermi's golden rule, the current is given by

$$
\begin{aligned}
J(F)= & \sum_{l>0} l \frac{e}{\tau_{0}} \sum_{n}\left[J_{n}\left(\frac{2 T_{1}}{e F d}\right) J_{n-l}\left(\frac{2 T_{1}}{e F d}\right)\right]^{2} \frac{1}{2 \pi^{2}} \\
& \times \int d^{2} k\left[n_{F}\left(E_{k}\right)-n_{F}\left(E_{k}+l e F d\right)\right] .
\end{aligned}
$$

Here the term $\sum\left[J_{n} J_{n-l}\right]^{2}$ arises due to the spatial overlap of the Wannier-Stark functions, and the Fermi functions reflect our assumption regarding inscattering. The electron density per period is given by

$$
N_{2 \mathrm{D}}=\rho_{0} k_{B} T_{e} \ln \left[1+\exp \left(\frac{\mu}{k_{B} T_{e}}\right)\right],
$$

which relates $\mu$ to $N_{2 \mathrm{D}}$. Again, it is possible to generalize this approach to more realistic scattering mechanisms $[17,18]$.

Sequential tunneling. - In this approximation the phase information is lost after each tunneling event between adjacent wells. The scattering within a well is treated selfconsistently by solving for the spectral functions $A(\mathcal{E}, \mathbf{k})$; in this work we use the self-consistent Born approximation [19] for the self-energy. The transitions to neighboring wells are calculated in lowest order of the coupling yielding $[14,19,20]$,

$$
\begin{aligned}
J(F)= & \frac{e}{2 \pi^{2}} \int d^{2} k \int \frac{d \mathcal{E}}{2 \pi \hbar} T_{1}^{2} A(\mathcal{E}, \mathbf{k}) A(\mathcal{E}+e F d, \mathbf{k}) \\
& \times\left[n_{F}(\mathcal{E})-n_{F}(\mathcal{E}+e F d)\right] .
\end{aligned}
$$

The carrier density is given by

$$
N_{2 \mathrm{D}}=\frac{1}{2 \pi^{2}} \int d^{2} k \int \frac{d \mathcal{E}}{2 \pi} n_{F}(\mathcal{E}) A(\mathcal{E}, \mathbf{k}) .
$$

This approach gives quantitative agreement with experiments in weakly coupled structures when realistic models for impurity and interface scattering are employed [10,14].

The important issue to recognize is that these three approaches treat scattering, external field, and coupling within different approximations. MBC does not properly include field-induced localization because of its inherent assumption of extended states, WSH treats scattering in lowest order perturbation theory (in particular, there is no broadening of the states), and ST is explicitly lowest order in the interwell coupling. In contrast to these shortcomings, a full quantum transport theory, based on nonequilibrium Green functions [21], is able to treat scattering, electric field, and coupling on equal footing. Such an approach was performed in Ref. [13] using a basis of Wannier-Stark states and restricting the analysis to a high electron temperature. Here we work within the basis $\Psi_{n}(z)$ and consider the general situation which allows an analysis of transitions between the simplified approaches MBC, WSH, and ST.

Nonequilibrium Green functions $(N G F)$. - - Here the current and electron density are given by $[19,21]$

$$
\begin{aligned}
& J(F)=\frac{e}{2 \pi^{2}} \int d^{2} k \frac{2}{\hbar} \operatorname{Re}\left\{T_{1} G_{n+1, n}^{<}(t, t, \mathbf{k})\right\}, \\
& N_{2 \mathrm{D}}=\frac{1}{2 \pi^{2}} \int d^{2} k G_{n, n}^{<}(t, t, \mathbf{k}),
\end{aligned}
$$

where $G_{m, n}^{<}\left(t, t^{\prime}, \mathbf{k}\right)=i\left\langle a_{n}^{\dagger}\left(t^{\prime}, \mathbf{k}\right) a_{m}(t, \mathbf{k})\right\rangle$, and $a_{n}^{\dagger}(t, \mathbf{k})$ and $a_{n}(t, \mathbf{k})$ are the creation and annihilation operators for the state $\Psi_{n}(z) e^{i(\mathbf{k} \cdot \mathbf{r})} / A$ in well $n$. We also need the retarded Green function, $G_{m, n}^{\text {ret }}\left(t, t^{\prime}, \mathbf{k}\right)=-i \Theta(t-$ $\left.t^{\prime}\right)\left\langle\left\{a_{m}(t, \mathbf{k}), a_{n}^{\dagger}\left(t^{\prime}, \mathbf{k}\right)\right\}\right\rangle$, where $\{A, B\}$ denotes the anticommutator. In the stationary state the Green functions depend only on the time difference $\tau=t-t^{\prime}$, and we define the Fourier transformation via [21]

$$
\begin{aligned}
G_{m, n}(\mathcal{E}, \mathbf{k})= & \int d \tau e^{i[\mathcal{F}-e F d(n+m) / 2](\tau / \hbar)} \\
& \times G_{m, n}(t, t-\tau, \mathbf{k}) .
\end{aligned}
$$

Without scattering between the $\mathbf{k}$ states and at $T_{1}=0$ the Green functions are diagonal in the well index: $G_{m, n}^{\text {ret }}(\mathcal{E}, \mathbf{k})=\delta_{m, n} g_{n}^{\text {ret }}(\mathcal{E}, \mathbf{k})$ with the free particle Green 
function $g_{n}^{\text {ret }}(\mathcal{E}, \mathbf{k})=1 /\left(\mathcal{E}-E_{k}+i 0^{+}\right)$. The full Green function is then determined by the Dyson equation,

$$
\begin{aligned}
G_{m, n}^{\mathrm{ret}}(\mathcal{E}, \mathbf{k})= & g_{m}^{\mathrm{ret}}\left(\mathcal{E}+e F d \frac{m-n}{2}, \mathbf{k}\right) \\
\times & {\left[\delta_{m, n}+\sum_{l} \Sigma_{m, l}^{\mathrm{ret}}\left(\mathcal{E}+e F d \frac{l-n}{2}, \mathbf{k}\right)\right.} \\
& \left.\times G_{l, n}^{\mathrm{ret}}\left(\mathcal{E}+e F d \frac{l-m}{2}, \mathbf{k}\right)\right] .
\end{aligned}
$$

Within the self-consistent Born approximation for the scattering the self-energy can be written as

$\sum_{m, n}^{\mathrm{ret}}(\mathcal{E}, \mathbf{k})=\delta_{m, n} \tilde{\Sigma}_{n}^{\mathrm{ret}}(\mathcal{E}, \mathbf{k})+T_{1} \delta_{m+1, n}+T_{1} \delta_{m-1, n}$,

with $\tilde{\Sigma}_{n}^{\text {ret }}(\mathcal{E}, \mathbf{k})=N_{d} / A \sum_{\mathbf{k}^{\prime}} V^{2} G_{n, n}^{\text {ret }}\left(\mathcal{E}, \mathbf{k}^{\prime}\right)$. If the scattering term $\tilde{\Sigma}_{n}^{\text {ret }}$ is neglected, the solution corresponds to the Wannier-Stark states (5). On the other hand, neglecting the coupling $T_{1}$ gives the spectral functions used in the sequential tunneling model. Equations (13) and (14) are solved self-consistently for $G^{\text {ret }}$. Then $G^{<}$is calculated via the Keldysh equation [21],

$$
\begin{aligned}
G_{m, n}^{<}(\mathcal{E}, \mathbf{k})= & \sum_{m_{1}} G_{m, m_{1}}^{\mathrm{ret}}\left(\mathcal{E}+e F d \frac{m_{1}-n}{2}, \mathbf{k}\right) \\
& \times \tilde{\Sigma}_{m_{1}}^{<}\left[\mathcal{E}+e F d\left(m_{1}-\frac{m+n}{2}\right), \mathbf{k}\right] \\
& \times G_{m_{1}, n}^{\mathrm{adv}}\left(\mathcal{E}+e F d \frac{m_{1}-m}{2}, \mathbf{k}\right) .
\end{aligned}
$$

According to our general assumption about inscattering we replace $\tilde{\Sigma}_{m}^{<}(\mathcal{E}, \mathbf{k})$ by its equilibrium value $-2 i n_{F}(\mathcal{E}) \times$ $\operatorname{Im}\left\{\tilde{\Sigma}_{m}^{\text {ret }}(\mathcal{E}, \mathbf{k})\right\}$. Finally, the current and electron density are calculated via Eqs. (10) and (11). The extension of this model to more realistic scattering processes is straightforward by using the respective self-energies in Eqs. (14) and (15) and relaxing the assumption about inscattering, although the calculations become very tedious (see, e.g., Ref. [22] where NGF has been applied to the resonant tunneling diode).

In Fig. 2 we display the evolution of the current-field relations for the different models from weakly to strongly coupled superlattices. The curves for MBC, ST, and NGF are qualitatively similar for all couplings. For low electric fields the current increases linearly with the electric field. Then there is a peak at intermediate fields, and negative differential conductivity occurs at higher field. For small $T_{1}$ as well as for high fields the result from ST is in quantitative agreement with the NGF result, while the results deviate for larger $T_{1}$. In contrast, the result from MBC is in quantitative agreement with the NGF result for large $T_{1}$ and small $e F d$. The WSH result diverges for $e F d \rightarrow 0$ [17], but approaches the NGF result for

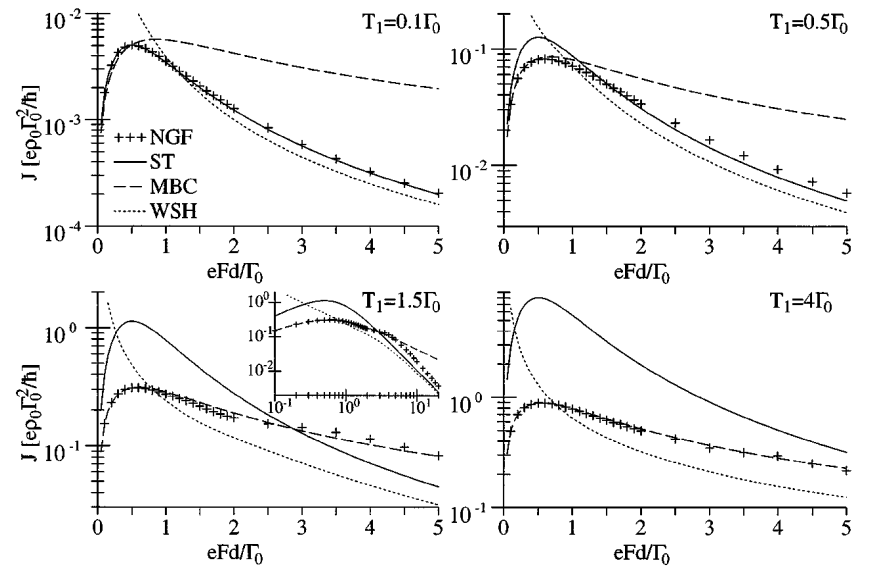

FIG. 2. Current-field relations calculated from nonequilibrium Green functions (NGF) in comparison with the standard approaches for $N_{2 \mathrm{D}}=0.2 \Gamma_{0} \rho_{0}$ and $k_{B} T_{e}=0.2 \Gamma_{0}$. For $T_{1}=$ $1.5 \Gamma_{0}$ the current-field relation is also shown over a wider field range in the inset. Here, one can see explicitly that the NGF result leaves the MBC curve for $e F d \gtrsim T_{1}$ and approaches the ST and WSH curves for large fields.

large fields. These results as well as further calculations for $k_{B} T_{e}<\Gamma, N_{2 \mathrm{D}}<\rho_{0} \Gamma$ are summarized by Fig. 1, depicting the respective regions in parameter space, where the different approaches approximate the NGF result.

Now we want to justify these ranges of validity by studying the quantum mechanical correlation between the wells $n$ and $m$ given by the retarded Green function. For a constant scattering self-energy $\tilde{\Sigma}_{n}^{\text {ret }}(\mathcal{E}, \mathbf{k})=-i \Gamma / 2$, of Eqs. (13) and (14), we have found an analytic solution

$$
G_{m, n}^{\mathrm{ret}}(\mathcal{E}, \mathbf{k})=\sum_{\alpha} \frac{J_{m-\alpha}\left(\frac{2 T_{1}}{e F d}\right) J_{n-\alpha}\left(\frac{2 T_{1}}{e F d}\right)}{\mathcal{E}-e F d\left(\frac{m+n}{2}-\alpha\right)-E_{k}+i \frac{\Gamma}{2}},
$$

which is a superposition of broadened Wannier-Stark states. The Wannier-Stark ladder becomes resolved if $e F d \gg \Gamma$. This defines the region of validity for the WSH approach, as indicated by the right region in Fig. 1. By Fourier transforming we obtain

$$
\begin{aligned}
G_{m, n}^{\mathrm{ret}}(t, t-\tau, \mathbf{k})= & -i \Theta(\tau) i^{n-m} e^{i\left\{[(m+n) / 2] e F d-E_{k}\right\}(\tau / \hbar)} \\
& \times e^{-\Gamma \tau / 2 \hbar} J_{m-n}\left[\frac{4 T_{1}}{e F d} \sin \left(\frac{e F d}{2 \hbar} \tau\right)\right] .
\end{aligned}
$$

Here the terms $G_{n \pm 1, n}^{\text {ret }}$ become of the order of $G_{n, n}^{\text {ret }}$ when $\left|J_{0}\right| \approx\left|J_{ \pm 1}\right|$, i.e., $\left|4 T_{1} /(e F d) \sin (e F d \tau / 2)\right| \approx \sqrt{2}$. This can be used as an estimate for the boundary between localization and delocalization. Because of the exponential factor in Eq. (17) only $\tau<2 \hbar / \Gamma$ is of relevance. If $e F d>\Gamma$ the magnitude of the sine takes the average value $\approx 1 / \sqrt{2}$. Then we find $2\left|T_{1}\right| \approx e F d$. If, on the other hand, $e F d<\Gamma$ we may replace $\sin (x) \approx x$ and have $2\left|T_{1}\right| \approx \Gamma$ at the time $\tau=\sqrt{2} \hbar / \Gamma$. From these estimates we conclude that the states are essentially delocalized if $2\left|T_{1}\right| \gg \Gamma$ and $2\left|T_{1}\right| \gg e F d$. In this case the 

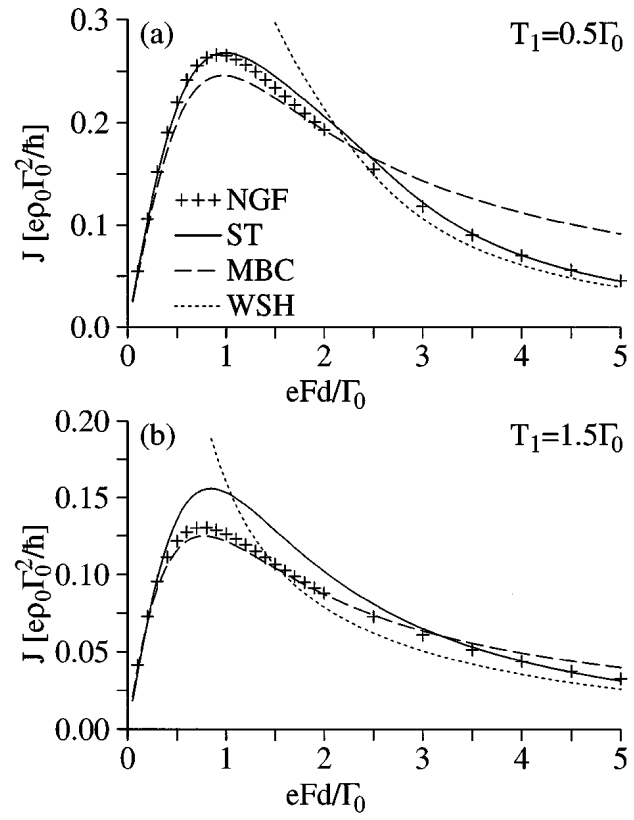

FIG. 3. Current-field relations for (a) high electron density $\left(N_{2 \mathrm{D}}=2 \Gamma_{0} \rho_{0}\right.$ and $\left.k_{B} T_{e}=0.2 \Gamma_{0}\right)$ and (b) high electron temperature $\left(k_{B} T_{e}=3 \Gamma_{0}\right.$ and $\left.N_{2 \mathrm{D}}=0.2 \Gamma_{0} \rho_{0}\right)$ for the different approaches. Note that the MBC result deviates from the NGF result at $e F d \gtrsim N_{2 \mathrm{D}} / \rho_{0}$ in (a).

miniband states form a useful basis as indicated in the upper left part of Fig. 1. On the other hand, for $2\left|T_{1}\right| \ll \Gamma$ or $2\left|T_{1}\right| \ll e F d$ the correlation functions $G_{m, n}^{\text {ret }}$ vanish for $m \neq n$ and the states are essentially localized so that the sequential limit can be used, as indicated in the lower part of Fig. 1.

For larger electron densities the agreement between the different approaches becomes better as shown in Fig. 3(a). These results together with further calculations indicate that ST is also valid if $N_{2 \mathrm{D}} / \rho_{0} \gtrsim 2\left|T_{1}\right|$, and $\mathrm{MBC}$ is also valid if $N_{2 \mathrm{D}} / \rho_{0} \gtrsim \Gamma$ and $N_{2 \mathrm{D}} / \rho_{0} \gtrsim e F d$. A similar trend is found for higher electron temperatures [Fig. 3(b)]. This agrees with the analytic findings of Ref. [13] where it is shown that NGF gives the same result as MBC in the limit $k_{B} T_{e} \gg\left|T_{1}\right|, e F d$.

In conclusion, we have explicitly shown that a transport calculation based on nonequilibrium Green functions contains the simple approaches MBC, WSH, and ST as limiting cases. For low temperature and low electron density the ranges of validity of the simplified approaches are depicted in Fig. 1, while for higher electron densities or temperatures, these ranges are enlarged. The essential message of our analysis is that for wide regions in parameter space (but not everywhere) a simplified theory can be found, which approximates the full theory satisfactorily. This should have important consequences for practical device modeling, where other complications, such as realistic scattering mechanisms, must be considered as well.

A. W. acknowledges financial support by the Deutsche Forschungsgemeinschaft.

*Present address: Institut für Theoretische Physik, Technische Universität Berlin, Hardenbergstrasse 36, 10623 Berlin, Germany.

[1] L. Esaki and R. Tsu, IBM J. Res. Dev. 14, 61 (1970).

[2] A. Sibille, J. F. Palmier, H. Wang, and F. Mollot, Phys. Rev. Lett. 64, 52 (1990).

[3] H. T. Grahn, R. J. Haug, W. Müller, and K. Ploog, Phys. Rev. Lett. 67, 1618 (1991).

[4] C. Waschke et al., Phys. Rev. Lett. 70, 3319 (1993).

[5] M. Holthaus, Phys. Rev. Lett. 69, 351 (1992).

[6] B. J. Keay et al., Phys. Rev. Lett. 75, 4102 (1995).

[7] P. A. Lebwohl and R. Tsu, J. Appl. Phys. 41, 2664 (1970).

[8] R. Tsu and G. Döhler, Phys. Rev. B 12, 680 (1975).

[9] D. Miller and B. Laikhtman, Phys. Rev. B 50, 18426 (1994).

[10] A. Wacker and A.-P. Jauho, Phys. Scr. T69, 321 (1997).

[11] A. Ya. Shik, Fiz. Tekh. Poluprovodn. 8, 1841 (1974) [Sov. Phys. Semicond. 8, 1195 (1975)].

[12] R. Tsu and L. Esaki, Phys. Rev. B 43, 5204 (1991).

[13] B. Laikhtman and D. Miller, Phys. Rev. B 48, 5395 (1993).

[14] A. Wacker, in Theory of Transport Properties of Semiconductor Nanostructures, edited by E. Schöll (Chapman and Hall, London, 1998) [cond-mat/9701105].

[15] A. A. Ignatov, E. P. Dodin, and V. I. Shashkin, Mod. Phys. Lett. B 5, 1087 (1991).

[16] X. L. Lei, N. J. M. Horing, and H. L. Cui, Phys. Rev. Lett. 66, 3277 (1991).

[17] S. Rott, N. Linder, and G. H. Döhler, Superlattices Microstruct. 21, 569 (1997).

[18] V. V. Bryksin and P. Kleinert, J. Phys. Condens. Matter 9, 7403 (1997).

[19] G. D. Mahan, Many-Particle Physics (Plenum, New York, 1990).

[20] S. Q. Murphy, J. P. Eisenstein, L. N. Pfeiffer, and K. W. West, Phys. Rev. B 52, 14825 (1995).

[21] H. Haug and A.-P. Jauho, Quantum Kinetics in Transport and Optics of Semiconductors (Springer, Berlin, 1996).

[22] R. Lake, G. Klimeck, R. C. Bowen, and D. Jovanovic, J. Appl. Phys. 81, 7845 (1997). 